\title{
A numerical and experimental investigation of the performance of sound intensity probes at high frequencies
}

\author{
Jacobsen, Finn; Cutanda, Vicente; Juhl, Peter Møller
}

Published in:

Acoustical Society of America. Journal

Link to article, DOI:

10.1121/1.421212

Publication date:

1998

Document Version

Publisher's PDF, also known as Version of record

Link back to DTU Orbit

Citation (APA):

Jacobsen, F., Cutanda, V., \& Juhl, P. M. (1998). A numerical and experimental investigation of the performance of sound intensity probes at high frequencies. Acoustical Society of America. Journal, 103(2), 953-961. https://doi.org/10.1121/1.421212

\section{General rights}

Copyright and moral rights for the publications made accessible in the public portal are retained by the authors and/or other copyright owners and it is a condition of accessing publications that users recognise and abide by the legal requirements associated with these rights.

- Users may download and print one copy of any publication from the public portal for the purpose of private study or research.

- You may not further distribute the material or use it for any profit-making activity or commercial gain

- You may freely distribute the URL identifying the publication in the public portal 


\title{
A numerical and experimental investigation of the performance of sound intensity probes at high frequencies
}

\author{
Finn Jacobsen, Vicente Cutanda, ${ }^{\text {a) }}$ and Peter M. Juhl \\ Department of Acoustic Technology, Technical University of Denmark, Building 352, DK-2800 Lyngby, \\ Denmark
}

(Received 18 February 1997; accepted for publication 5 November 1997)

\begin{abstract}
The influence of scattering and diffraction on the performance of sound intensity probes has been examined using a boundary element model of an axisymmetric two-microphone probe with the microphones in the usual face-to-face arrangement. On the basis of calculations for a variety of sound field conditions and probe geometries it is concluded that the optimum length of the spacer between the microphones is about one microphone diameter; with this geometry the effect of diffraction and the finite difference error almost counterbalance each other up to about an octave above the frequency limit determined by the finite difference approximation. This seems to be valid under virtually any sound field condition that could be of practical importance in sound power determination. The upper frequency limit corresponds to about $10 \mathrm{kHz}$ for an intensity probe with $\frac{1}{2}$-in. microphones, which means that it should be possible to cover most of the audible frequency range, say, from $50 \mathrm{~Hz}$ to $10 \mathrm{kHz}$, with a single probe configuration. The numerical results have been confirmed by a series of experiments. (c) 1998 Acoustical Society of America.
\end{abstract}

[S0001-4966(98)04202-7]

PACS numbers: 43.58.Fm, 43.50.Yw, 43.38.Kb [SLE]

\section{INTRODUCTION}

All sound intensity probes in commercial production today are based on the "two-microphone" principle which employs two closely spaced pressure microphones. ${ }^{1}$ One of the fundamental limitations of this measurement principle is due to the approximation of the particle velocity by a difference of pressures at two closely spaced points. This finite difference approximation obviously imposes an upper frequency limit. The interference of the microphones on the sound field also imposes an upper frequency limit. One of the results of the many investigations in the late 1970s and early 1980s was that the "face-to-face" arrangement is advantageous in this respect. ${ }^{2,3}$ In this configuration the microphones are mounted against each other with a solid plug, a "spacer," between the sensing parts, which means that the bulk of the probe in effect is a cylindrical body.

In the past decade research in this field has concentrated on problems at low and medium frequencies, and the most significant improvement of the instrumentation has been the development of microphones with reduced production tolerances of the phase characteristics and very low vent sensitivity. ${ }^{4}$ The purpose of this paper is to examine whether the performance of intensity probes could be improved at high frequencies.

\section{HIGH-FREQUENCY LIMITATIONS}

\section{A. The finite difference approximation}

Although the finite difference approximation error in principle depends on the sound field, ${ }^{5-7}$ practice has shown

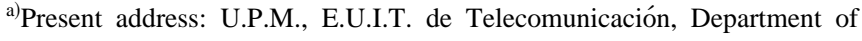
Audiovisual Engineering and Communications, Ctr de Valencia km 7, E28031 Madrid, Spain.
}

that the influence of the error on measurement of sound power can be conservatively predicted from the expression for intensity estimation in a plane wave with a direction that coincides with the axis of the probe, ${ }^{1}$

$$
\hat{I}_{r}=I_{r} \frac{\sin k \Delta r}{k \Delta r}
$$

where $\hat{I}_{r}$ is the estimate of the true intensity component $I_{r}, k$ is the wave number, and $\Delta r$ is the microphone separation distance. This expression, which is shown in Fig. 1, implies that the condition

$$
k \Delta r<1.15
$$

ensures that the error is less than $1 \mathrm{~dB}$, corresponding to an upper frequency limit of about $5 \mathrm{kHz}$ if the separation distance is $12 \mathrm{~mm}$, which has usually been regarded as the minimum distance to give acceptably small diffraction errors with standard $\frac{1}{2}$-in. condenser microphones. This combination is very common. One can, of course, use smaller microphones separated by a correspondingly shorter distance; with $\frac{1}{4}$-in. microphones separated by a 6-mm spacer, for example, the upper frequency limit will be about $10 \mathrm{kHz}$, which is more acceptable. Unfortunately the influence of several other measurement errors, of which the most well known and most serious is phase mismatch, is inversely proportional to the length of the spacer. ${ }^{5,8-10}$ This means that an intensity probe with smaller microphones separated by a shorter distance will have a higher lower limiting frequency. To this can be added that, quite apart from the influence of the separation distance, $\frac{1}{4}$-in. microphones are considerably more noisy than $\frac{1}{2}$-in. microphones, they are difficult to use because of their low capacitance, and they are not commercially available in pairs as well matched as $\frac{1}{2}$-in. microphones are. 


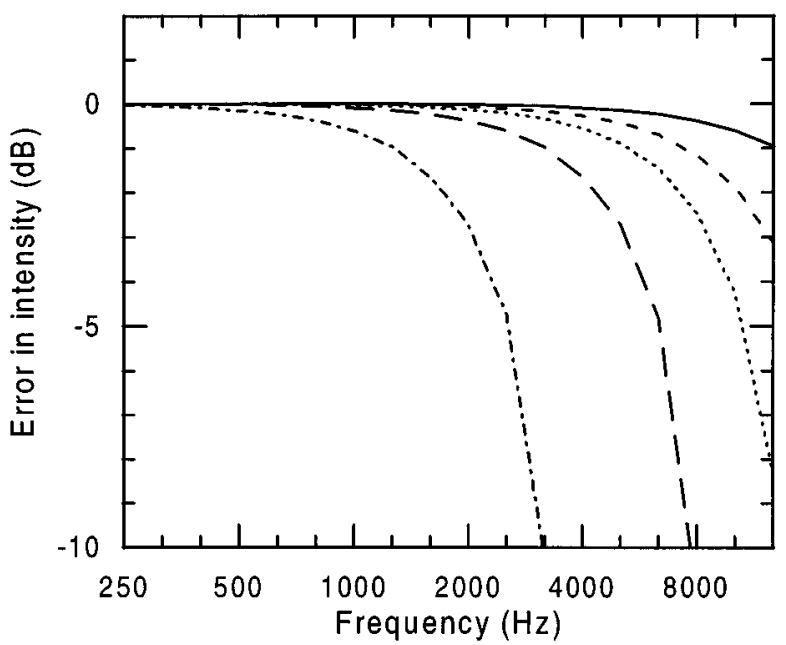

FIG. 1. Finite difference approximation error in plane wave of axial incidence for different spacer lengths; — , $5 \mathrm{~mm} ;---, 8.5 \mathrm{~mm} ; \cdots, 12 \mathrm{~mm}$; ---, $20 \mathrm{~mm} ;-\cdot-\cdot, 50 \mathrm{~mm}$.

Another way of overcoming the relatively low upper frequency limit of the sound intensity technique might be to "correct" the finite difference error by multiplying with a factor of $k \Delta r / \sin k \Delta r$, as suggested by Balant et al. ${ }^{11}$ However, this cannot be recommended in the general case; it tends to lead to overestimation because the intensity vector will usually not be perpendicular to the measurement surface used in sound power determination.

One can also extend the frequency range by combining measurements with a probe with $\frac{1}{2}$-in. microphones separated by a relatively long spacer with measurements with $\frac{1}{4}$-in. microphones and a 6-mm spacer-but that is not an attractive solution.

\section{B. Scattering and diffraction effects}

The foregoing considerations seem to be based on the assumption that the intensity probe actually measures the sound pressure at two discrete positions as it would be in the absence of the probe. However, because of the interference of the probe on the sound field, that is the case only at low frequencies.

Scattering and diffraction effects have been examined experimentally by many workers in the area. ${ }^{2,3,12-17}$ All these investigations have been restricted to examining the performance of intensity probes of various configurations in the simplest of all sound fields, a plane progressive wave, though; and in most cases only axial incidence has been considered. A general conclusion is that the face-to-face configuration with a solid spacer between the microphones is advantageous. With the microphones arranged in this configuration the phase difference between the two microphone signals increases nearly linearly with the frequency over a wider frequency range than with the same configuration without the spacer or with any other configuration, in particular for nonaxial incidence of the plane wave. It has also been observed that a long cylindrical probe (which cannot be used in practice) performs better than a similar short probe in this respect. ${ }^{16}$ (a)

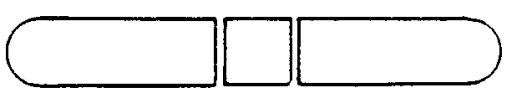

(b)

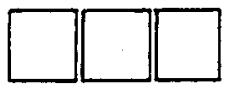

FIG. 2. Intensity probe geometries. (a) Long probe, (b) short probe.

One of the characteristics of the face-to-face arrangement with a spacer between the microphones is that there is a small cavity in front of the diaphragm of each microphone. This cavity is connected to the outside via the slots of the microphone grid. The experimental results published in Refs. 15 and 16 imply that diffraction effects due to the cylinder in combination with the resonance of the cavities give rise to a pressure increase on both diaphragms that amounts to about $5 \mathrm{~dB}$ at $10 \mathrm{kHz}$ for a probe with $\frac{1}{2}$-in. microphones subjected to a plane wave of axial incidence. Watkinson and Fahy suggest that this increase could be used to compensate for the finite difference error. ${ }^{15}$

Diffraction experiments are rather difficult to perform; they tend to be disturbed by the influence of supporting devices, imperfections of the anechoic room, etc. Therefore, numerical models would seem to have some advantages in studying the performance of various probe configurations. However, only one single numerical investigation of the behavior of sound intensity probes has been found in the literature. ${ }^{18}$ Also here the analysis was restricted to plane waves of axial incidence. Unfortunately, the face-to-face arrangement with a spacer between the microphones, which seems to be superior according to the experimental data presented in Refs. 2, 3, and 14-16, was not examined in this investigation.

It must be concluded that diffraction effects have been studied systematically only in very simple sound fields, and that existing sound intensity probes have not been optimized for high-frequency performance.

\section{A BOUNDARY ELEMENT MODEL}

The boundary element method based on the Helmholtz integral equation is particularly suited for solving scattering and diffraction problems. ${ }^{19,20}$ Such a model has been implemented for various axisymmetric probe configurations, two of which are shown in Fig. 2. Details of the model of intensity probes have been given elsewhere, ${ }^{21}$ here it suffices to say that each pressure signal is calculated as a weighted average of the pressure on the diaphragm, the weighting function being due to the shape of the dominating first membrane mode and the finite size of the backplate behind the diaphragm. $^{22}$ The model is idealized in three respects: (i) nonaxisymmetric parts of the probe are ignored, (ii) a parabolic mode shape of the diaphragm is assumed, and (iii) the finite acoustic impedance of the diaphragm is not taken into 


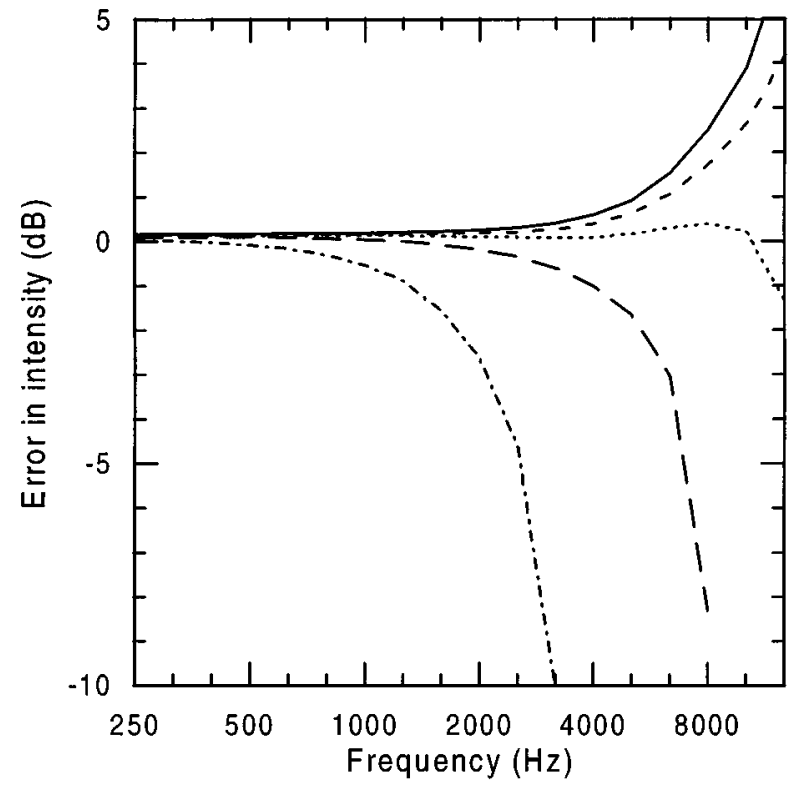

FIG. 3. Error of short probe with half-inch microphones in a plane wave of axial incidence. Length of spacer: ——, $5 \mathrm{~mm} ;---, 8.5 \mathrm{~mm} ; \cdots 12 \mathrm{~mm}$; ,$--- 20 \mathrm{~mm} ;-\cdot-\cdot \cdot, 50 \mathrm{~mm}$.

account. The two last mentioned simplifications are certainly reasonable in the frequency range of concern here, well below the resonance frequency of the diaphragm. ${ }^{22}$ The first one, which leads to a significant reduction of the required computer memory and calculation time, is perhaps also reasonable since the diffraction behavior of existing probes with $\frac{1}{2}$-in. microphones can be expected to be dominated by the bulk of the microphones and the spacer.

All results presented in the following have been calculated for $\frac{1}{2}$-in. microphones, that is, for a cylinder with a diameter of $12.7 \mathrm{~mm}$. There is a gap of $1.1 \mathrm{~mm}$ between the diaphragm and the solid spacer. The diameter of the diaphragm is $9 \mathrm{~mm}$, and the diameter of the backplate is 7.2 $\mathrm{mm}$. Two geometries are investigated here, a "long probe", of which each "microphone" is a 31.8-mm-long cylinder with a hemispherical end, and a "short probe," the microphones of which are $12 \mathrm{~mm}$ long and have flat ends. (Various other geometries have also been examined.) The geometry of the short probe is actually modeled after a probe in commercial production, Brüel \& Kjær's type 3548 with 4181 microphones. Note that "length of spacer" includes one gap (i.e., half a gap at both ends), so the physical length of a " 12 -mm spacer" is $10.9 \mathrm{~mm}$. The calculations have been carried out at the one-third octave center frequencies from $250 \mathrm{~Hz}$ to $12.5 \mathrm{kHz}$. Evidently, reducing all dimensions of the probe by a factor of 2 corresponds to doubling the frequency.

\section{A. Numerical results; single position}

Figure 3 shows the indicated sound intensity normalized with the true intensity, calculated for the short probe with five different spacers in a plane wave of axial incidence. The intensity has been determined from the two (complex) pressure signals as sensed by the probe, $\hat{p}_{1}$ and $\hat{p}_{2}$, as follows:

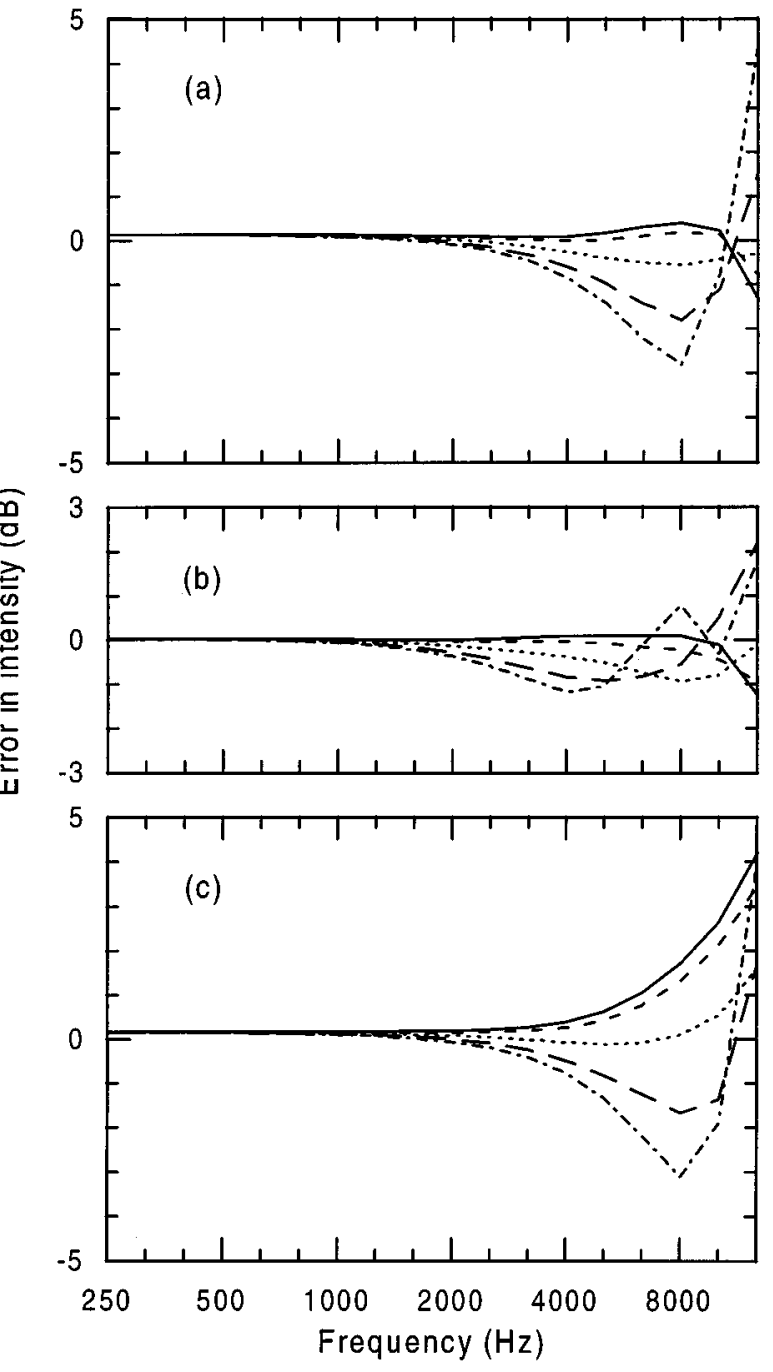

FIG. 4. Errors of intensity probes with $\frac{1}{2}$-in. microphones. (a) Short probe, 12-mm spacer, (b) long probe, 12-mm spacer, (c) short probe, $8.5-\mathrm{mm}$ spacer. Angle of incidence:,$- 0^{\circ} ;---, 20^{\circ} ; \cdots, 40^{\circ} ;--, 60^{\circ} ;-\cdot-\cdot$, $80^{\circ}$.

$$
\hat{I}_{r}=\frac{\operatorname{Im}\left\{\hat{p}_{1} \hat{p}_{2}^{*}\right\}}{2 \rho c k \Delta r},
$$

where $\rho$ is the density of air. At high frequencies the performance of the probe is essentially the result of the combined effect of the finite difference error shown in Fig. 1 and the pressure increase mentioned above, and it is apparent that the two effects very nearly counterbalance each other up to 10 $\mathrm{kHz}$ with the $12-\mathrm{mm}$ spacer, as anticipated by Watkinson and Fahy. ${ }^{15}$ A shorter spacer leads to overestimation, and with a long spacer the upper frequency limit is determined by the sinc-function, Eq. (1). Results obtained with the long probe are quite similar. It is interesting that a probe with $\frac{1}{2}$-in. microphones separated by a $12-\mathrm{mm}$ spacer performs better at high frequencies than a probe with $\frac{1}{4}$-in. microphones separated by a 12-mm spacer; this follows from the fact that the compensating pressure increase is shifted upwards by an octave in the latter case.

Figure 4 shows the errors for nonaxial incidence, calculated for the three different combinations of probe shapes 

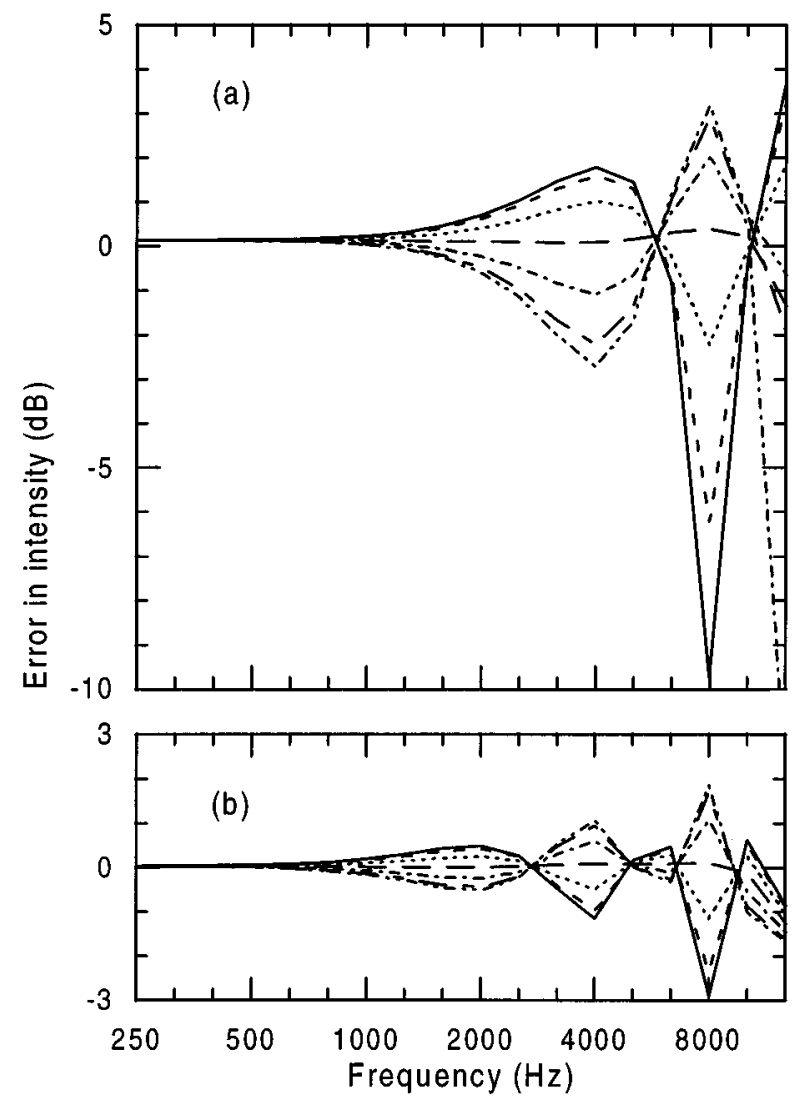

FIG. 5. Errors of intensity probes with half-inch microphones and a 12-mm spacer in an axial standing wave field with a standing wave ratio of $24 \mathrm{~dB}$. (a) Short probe, (b) long probe. Phase angle between the two interfering plane waves:,$--90^{\circ} ;---,-60^{\circ} ; \cdots,-30^{\circ} ;--, 0^{\circ} ;-\cdot-\cdot, 30^{\circ}$; ,$---- 60^{\circ} ;-\cdots-\cdots, 90^{\circ}$.

and spacer lengths. The figure demonstrates that the errors increase with the angle of incidence if the length of the spacer is optimized for axial incidence; they are much larger if the plane wave propagates in a direction almost perpendicular to the probe. With a shorter spacer the influence of the angle of incidence is increased. It can also be seen that the sharp edges of the short probe have an unfavorable influence for nonaxial incidence; the longer probe with rounded ends is clearly superior. Note that the short probe overestimates the intensity slightly at low frequencies irrespective of the angle of incidence, in agreement with the experimental observation that the "effective separation distance"' is slightly larger than the actual distance. ${ }^{16}$

Finally Fig. 5 demonstrates that the errors vary with the position of the probe in a standing wave field. It is interesting and rather surprising that, irrespective of the standing wave ratio, the effect of diffraction is the same both at pressure maxima and minima as in a plane progressive wave, whereas diffraction has a more serious influence and increases with the standing wave ratio at positions midway between these positions. (See the Appendix.) It is also apparent that the longer the probe, the less the error.

The influence of the gap in front of each microphone diaphragm has also been examined; the gap has very little influence.

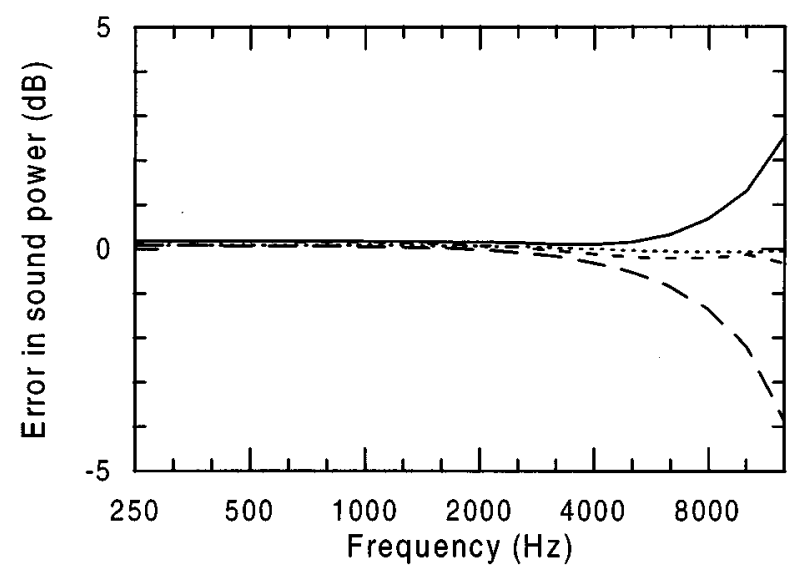

FIG. 6. Calculated error in sound power determination with the short probe in the absence of extraneous noise. —,$\frac{1}{2}$-in. microphones, 8.5-mm spacer; ---, $\frac{1}{2}$-in. microphones, 12-mm spacer; $\cdots, \frac{1}{4}$-in. microphones, 6-mm spacer; --, $\frac{1}{4}$-in. microphones, 12-mm spacer.

\section{B. Numerical results; sound power determination}

The most important application of sound intensity measurements is the determination of sound power, which involves integrating the normal component of the intensity over a surface that encloses the source under test. To examine the influence of scattering and diffraction on sound power determination, such measurements have been simulated by summing over the indicated intensity at a number of positions on a surface that encloses a monopole source in free field, with or without another monopole source outside the surface. The surface is a cube of $1 \times 1 \times 1 \mathrm{~m}$, and the normal component of the intensity is calculated at 36 points on each face of the surface. The calculations have been made for the short probe with $\frac{1}{2}$-in. microphones and various spacers as described in the foregoing, but also for a similar probe with $\frac{1}{4}$-in. microphones. (The latter is no longer a mere scaling, because the measurement surface is fixed.)

In Fig. 6 are shown the results of such a simulated sound power measurement without an extraneous source. As can be seen the errors are more or less as one would expect from Fig. 3, that is, in spite of the finite difference error the performance of a probe with $\frac{1}{2}$-in. microphones and a 12-mm spacer is nearly perfect up to $12.5 \mathrm{kHz}$, whereas the sound power is overestimated with a shorter spacer. With a probe with $\frac{1}{4}$-in. microphones and a $12-\mathrm{mm}$ spacer the finite difference error dominates below $12.5 \mathrm{kHz}$; therefore the sound power is underestimated.

Figure 7 shows the results of similar "measurements", in the presence of background noise from an uncorrelated monopole source. "Uncorrelated" implies that the calculated sound intensity component due to the disturbing source at each position has been added to the calculated sound intensity component due to the primary source. (If both sources were included in one boundary element calculation the result would correspond to completely correlated sources, which generate an interference field.) In the results presented in Fig. 7(a) and (b) the extraneous source is $2 \mathrm{~m}$ from the center of one of the faces of the cube, and its sound 

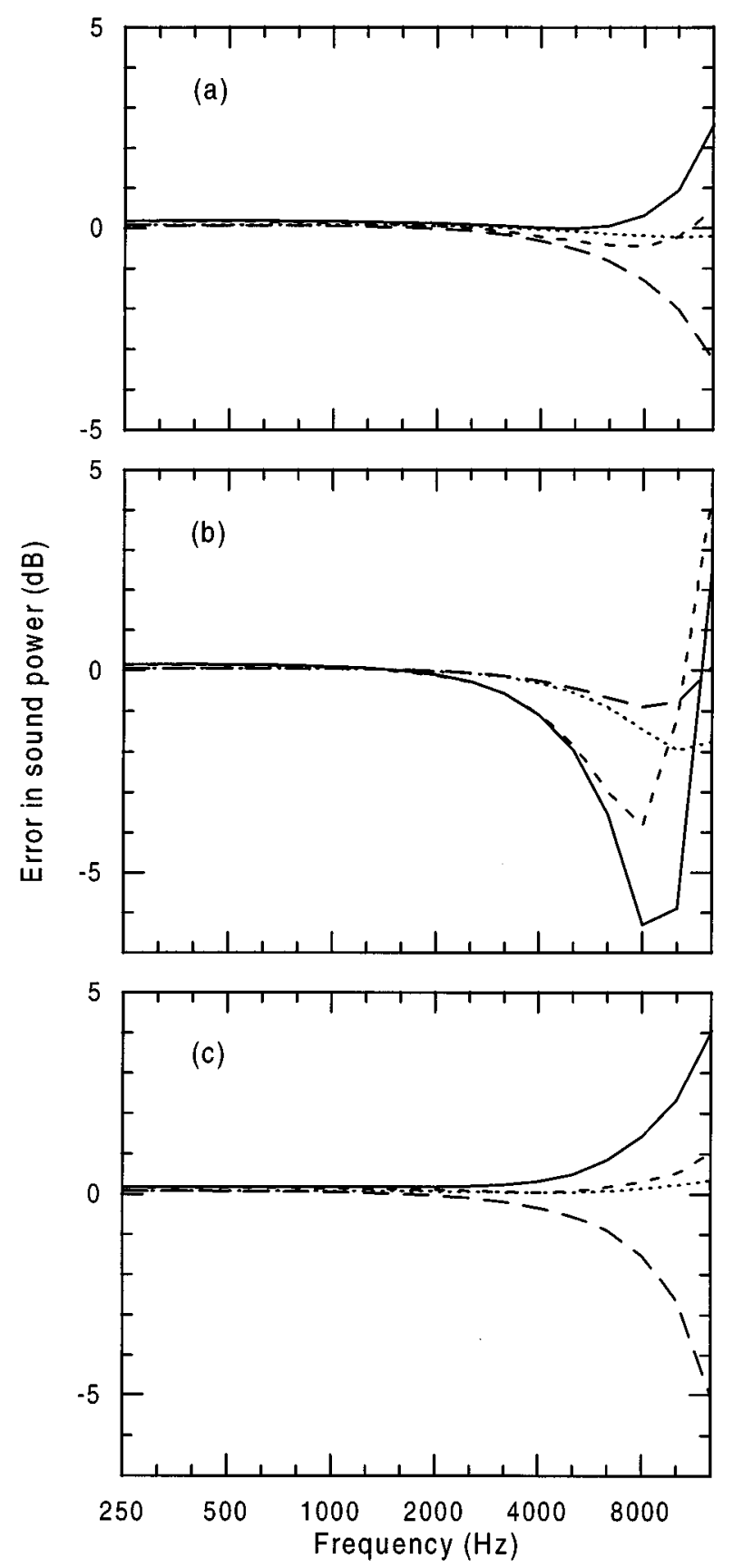

FIG. 7. Calculated error in sound power determination with the short probe in the presence of background noise from an uncorrelated source. (a) Extraneous source $10 \mathrm{~dB}$ stronger than the source under test, placed $2 \mathrm{~m}$ from one of the faces of the cubic measurement surface; (b) extraneous source $20 \mathrm{~dB}$ stronger than the source under test, placed $2 \mathrm{~m}$ from one of the faces of the cubic measurement surface; (c) extraneous source $20 \mathrm{~dB}$ stronger than the source under test, placed diagonally $2 \mathrm{~m}$ from an edge of the cubic measurement surface. $\longrightarrow, \frac{1}{2}$-in. microphones, 8.5 -mm spacer; ---, $\frac{1}{2}$-in. microphones, 12-mm spacer; $\cdots, \frac{1}{4}$-in. microphones, 6-mm spacer; --, $\frac{1}{4}$-in. microphones, $12-\mathrm{mm}$ spacer.

power outputs are, respectively, 10 and $20 \mathrm{~dB}$ larger than the sound power of the source under test. It is apparent that the intensity probe with $\frac{1}{2}$-in. microphones and a $12-\mathrm{mm}$ spacer is able to suppress noise from an extraneous source only up to a certain level at high frequencies, the reason being that the surface integral of the intensity associated with the dis- turbing source differs from zero because of diffraction effects. On the other hand, Fig. 7(c) demonstrates that the ability of suppressing disturbing noise depends on the position of the extraneous source. In this case the source is in a plane that bisects the cubic measurement surface diagonally, $2 \mathrm{~m}$ from the nearest edge, and although its sound power output is again $20 \mathrm{~dB}$ larger than the sound power of the source under test, the performance of the various combinations of probes and spacers is almost unaffected by the strong disturbing sound field. The influence of the position of the extraneous source on the performance of a probe with $\frac{1}{2}$-in. microphones and an $8.5-\mathrm{mm}$ spacer is particularly strong, in agreement with the observation that this configuration is more seriously affected by the angle of incidence of a plane wave.

\section{DISCUSSION}

The numerical results presented in the foregoing lead to the conclusion that the optimum length of the spacer is about $12 \mathrm{~mm}$ for a sound intensity probe with $\frac{1}{2}$-in. microphones in the face-to-face configuration, and that such a probe performs tolerably well up to $10 \mathrm{kHz}$ under a variety of sound field conditions. In other words, it covers just about the entire frequency range where sound power measurement is relevant. This is rather surprising in view of the fact that intensity probes that comply with this description have been on the market for about 15 years. ${ }^{23}$ If one consults Fahy's monograph on sound intensity and its measurement one certainly gets the impression that the upper frequency range of such a probe is $5 \mathrm{kHz}$ in accordance with the inequality (2). ${ }^{1}$ According to a well-known manufacturer of sound intensity probes, their probes should be used up to $5 \mathrm{kHz}$ with a $12-\mathrm{mm}$ spacer, ${ }^{24}$ and up to $6.3 \mathrm{kHz}$ with an $8.5-\mathrm{mm}$ spacer. ${ }^{25}$ Moreover, an IEC standard on instruments for the measurement of sound intensity actually requires that the sound intensity response of the probe in a plane progressive wave of axial incidence shall follow the sinc-function [Eq. (1)] within a certain tolerance. ${ }^{26}$

The most probable explanation of the remarkable fact that no one has discovered that the probe performs very well at much higher frequencies than $5 \mathrm{kHz}$ is that Brüel \& Kjær's sound intensity microphones of type 4181, which are rather dominating in this area, are overdamped, so-called free field microphones, that is, microphones designed to have a flat response in a plane wave of $0^{\circ}$ incidence. Since, surprisingly, the free field responses of two microphones in the face-to-face arrangement with a solid spacer between them differ little from the free field response of one microphone, as pointed out by Fahy and Elliott, ${ }^{3}$ this means that the pressure response of a probe with $\frac{1}{2}$-in. microphones of type B\&K 4181 in a plane wave of axial incidence is essentially flat up to $10 \mathrm{kHz}$; the intensity response, however, is not. The resulting underestimation may well have been confounded with finite difference errors. Clearly, "pressure" microphones rather than "free field" microphones should be used, as also pointed out by Fahy and Elliott. ${ }^{3}$ However, whereas one cannot in the general case compensate for the finite difference approximation error, it is unproblematic to compensate for a frequency-dependent pressure response. 
Both the IEC standard on sound intensity instruments ${ }^{26}$ and the corresponding American ANSI standard ${ }^{27}$ require a flat pressure response of the probe in a plane progressive wave of axial incidence (within a certain tolerance). However, a probe with $\frac{1}{2}$-in. microphones of the "pressure field" type does not have a flat pressure response above $5 \mathrm{kHz} .{ }^{16,21}$ It seems reasonable to optimize the high-frequency performance of sound intensity probes with respect to the intensity response rather than the pressure response, the more so since the pressure response at high frequencies depends strongly on the angle of incidence whereas it seems to be possible to obtain an intensity response that is essentially flat.

Although the resonances of the cavities compensate very well for the finite difference error in some cases, the compensation is not perfect, cf. Figs. 4 and 5. It is apparent that fairly large errors can occur, also below the frequency that has hitherto been believed to be the upper frequency limit of this configuration, when the angle between the axis of the probe and the direction of the (true) intensity is nearly $90^{\circ}$. In fact, other numerical experiments, not presented here, have shown that arbitrarily large errors can occur under sufficiently extreme sound field conditions, also well below 5 $\mathrm{kHz}$. However, this is unlikely to be a serious problem in sound power determination, because the component of the intensity in the direction of the probe is very small under such circumstances, which means that it does not contribute very much to the surface integral. This conclusion is supported by the simulated sound power measurements presented in Figs. 6 and 7: although errors occur in measurement of sound intensity at certain angles of incidence, they tend to be averaged out in sound power determination. In fact, it is easy to show that a plane wave generated by a distant extraneous source in free field would not in any way disturb sound power determination obtained by integrating the normal component of the intensity over a rectangular surface (in theory), irrespective of the relative strengths of the sources and irrespective of how much the sound field is disturbed by the presence of the intensity probe, provided that the probe is axisymmetric and symmetric about the spacer. (In practice the measurement accuracy would deteriorate because of spatial sampling errors if the extraneous sound field were sufficiently strong.)

For the same reason the shape of the intensity probe is probably not as important in sound power determination as Figs. 4 and 5 would seem to indicate. Although it is clear that the sharp edges of the short probe are unfavorable, it can be useful that the probe can be placed very near a vibrating surface, which means that it cannot be very long. A probe slightly longer than the "short" probe and with rounded ends would probably be the best compromise.

\section{EXPERIMENTAL RESULTS}

The most important conclusion to be drawn from the numerical results presented in the foregoing is that the optimum length of the spacer between the two microphones of an intensity probe is about one microphone diameter; with this configuration the useful frequency range of the probe is extended by about an octave above the limit determined by the finite difference error. In particular, a probe with $\frac{1}{2}$-in.

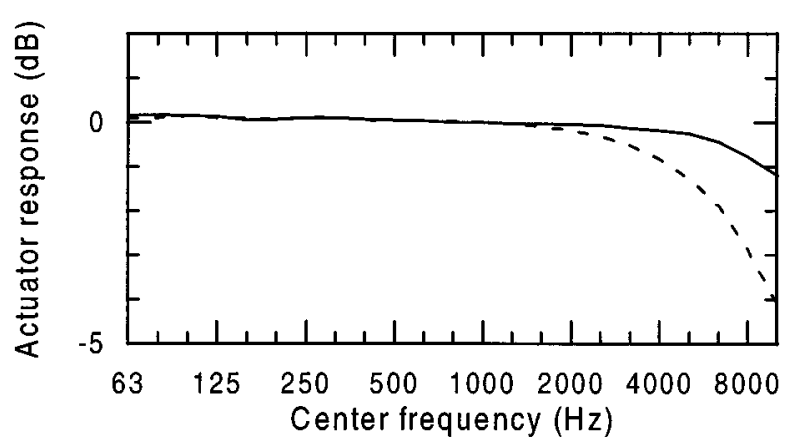

FIG. 8. Electrostatic actuator response of microphones of intensity probe. , B\&K type 4178 ; ---, B\&K type 4181.

microphones separated by a $12-\mathrm{mm}$ spacer should be able to cover the frequency range up to $10 \mathrm{kHz}$ in sound power determination with insignificant errors under any realistic sound field condition.

To test this conclusion a series of experiments has been carried out: the sound power of a loudspeaker driven with pink noise, $B \& K$ 4205, was determined under a variety of acoustic conditions. The source was placed on the floor, and the radiated sound power was estimated by scanning manually with an intensity probe over the five faces of a cubic surface of $1 \times 1 \times 1 \mathrm{~m}$.

A frequency analyzer of type $B \& K 3550$ was used in combination with an intensity probe of type B\&K 3548 , either with $\frac{1}{2}$-in. microphones of type B\&K 4181 or with $\frac{1}{4}$-in. microphones of type B\&K 4178. Since these microphones are so-called free-field microphones it is necessary to compensate for the drop of the pressure sensitivity at high frequencies. Figure 8 shows the pressure response of the two sets of microphones, determined with an electrostatic actuator. All the results presented in the following have been corrected with the corresponding actuator response. (In the frequency range well below the resonance frequency of the microphones the radiation impedance is much smaller than the acoustic impedance of the diaphragm, which means that the actuator response is proportional to the pressure response.)

The first series of measurements were carried out in a large $\left(240 \mathrm{~m}^{3}\right)$ reverberant room with a reverberation time of about $4 \mathrm{~s}$ under three conditions: (i) without extraneous noise, (ii) with strong diffuse background noise from a distant source (Airap A14 from Électricité de France), and (iii) with strong nondiffuse and diffuse background noise from the same source placed about $2.5 \mathrm{~m}$ from the surface. In the last mentioned case the partial sound power of the nearest $1-m^{2}$ segment was negative in the entire frequency range.

The measurements with $\frac{1}{4}$-in. microphones were carried out with a 6-mm spacer and with a $12-\mathrm{mm}$ spacer. The former measurement, which can be expected to be reliable at high frequencies, served as the reference in the frequency range from $4-10 \mathrm{kHz}$. The measurements with $\frac{1}{2}$-in. microphones were carried out with an $8.5-\mathrm{mm}$ spacer, a $12-\mathrm{mm}$ spacer, and a 50-mm spacer. In order to reduce the effect of transducer phase mismatch as far as possible, all measure- 

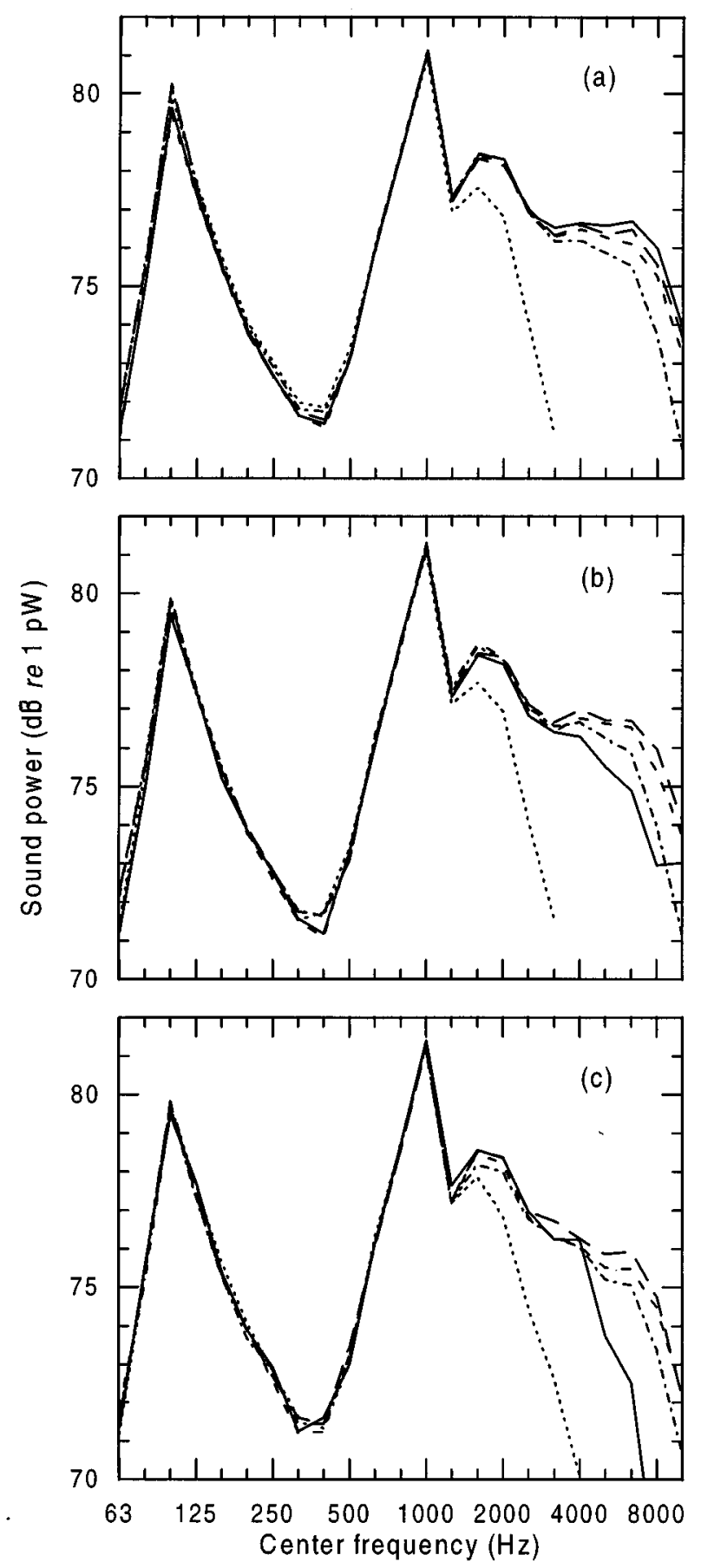

FIG. 9. Sound power of source in a reverberation room, measured with different combinations of microphones and spacers. (a) No extraneous noise, (b) strong diffuse background noise from an extraneous source, (c) strong diffuse and nondiffuse noise from an extraneous source. $-\frac{1}{2}$-in. microphones, 8.5 -mm spacer; ---, $\frac{1}{2}$-in. microphones, 12 -mm spacer; $\cdots, \frac{1}{2}$-in. microphones, 50-mm spacer; --, $\frac{1}{4}$-in. microphones, 6-mm spacer; -·-·, $\frac{1}{4}$-in. microphones, 12 -mm spacer.

ments were repeated with the two microphones interchanged. ${ }^{28}$

The results of the sound power measurements are presented in Fig. 9; Fig. 10, which shows the corresponding values of the pressure-intensity index, gives an impression of the acoustic conditions. It can be seen from Fig. 9 that prac-

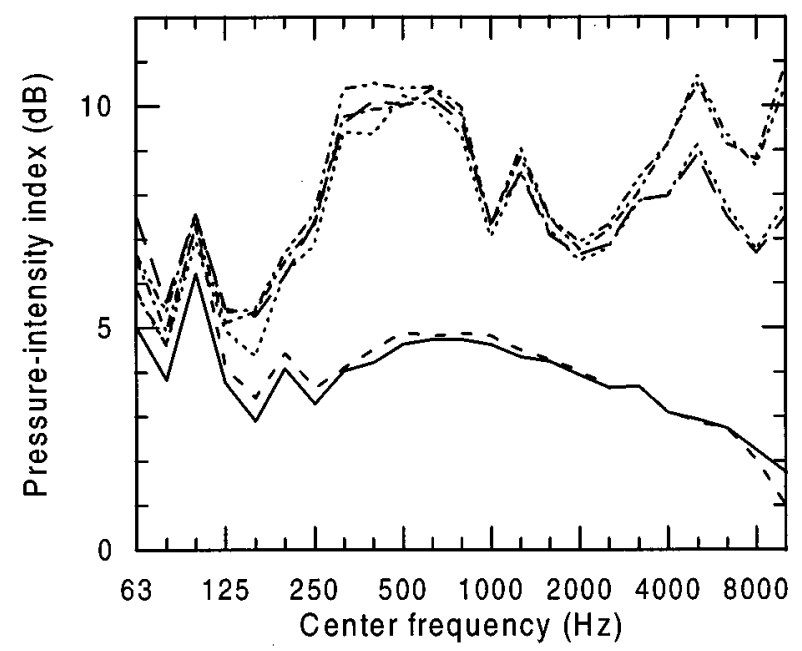

FIG. 10. Pressure-intensity index. $\longrightarrow, \frac{1}{4}$-in. microphones, 6-mm spacer, no extraneous noise; ---, $\frac{1}{2}$-in. microphones, 12 -mm spacer, no extraneous noise; $\cdots, \frac{1}{4}$-in. microphones, 6 -mm spacer, diffuse noise;,$-- \frac{1}{2}$-in. microphones, 12-mm spacer, diffuse noise; -.-·, $\frac{1}{4}$-in. microphones, 6-mm spacer, nondiffuse and diffuse noise;,$--- \frac{1}{2}$-in. microphones, 12-mm spacer, nondiffuse and diffuse noise.

tically all measurements are in agreement from $63 \mathrm{~Hz}$ to 1.25 $\mathrm{kHz}$. However, without compensation for phase mismatch significant errors occurred with the $\frac{1}{4}$-in. microphones in most of the frequency range. From $1.6 \mathrm{kHz}$ and upwards the combination of $\frac{1}{2}$-in. microphones and the 50 -mm spacer underestimates, but it is worth noting that the error is less than predicted by the idealized expression for an axial plane wave (Fig. 1), and that the size of the error depends on the sound field conditions, which leads to the conclusion that one cannot compensate for the finite difference error. The combination of $\frac{1}{4}$-in. microphones and a $12-\mathrm{mm}$ spacer leads to underestimation from $5 \mathrm{kHz}$ and upwards, more or less as expected. The measurements with $\frac{1}{2}$-in. microphones and the 12-mm spacer are in fair agreement with the reference measurements, confirming the predicted advantage of this combination. The behavior of the combination of $\frac{1}{2}$-in. microphones and the 8.5-mm spacer is more complicated. As can be seen, it overestimates slightly under mild measurement conditions, but underestimates under more difficult conditions. It seems as if the ability to suppress extraneous noise at high frequencies deteriorates if the spacer is significantly shorter than the diameter of the microphones, in agreement with the numerical results (cf. Fig. 7).

Figure 11 summarizes the high-frequency performance of the sound intensity probe with the most favorable geometry, a probe with $\frac{1}{2}$-in. microphones and a $12-\mathrm{mm}$ spacer, in sound power determination under the three acoustic conditions described above. Clearly the probe performs quite well even at 8 and $10 \mathrm{kHz}$.

Many other sound power measurements have been carried out, with similar results. It should finally be mentioned that no difference has been found between the performance of the B\&K 3548 probe and the newer, smoother, more robust version of the probe with an improved brace, UA $1250 .{ }^{25}$ Likewise, plastic cones mounted on the B\&K 3548 


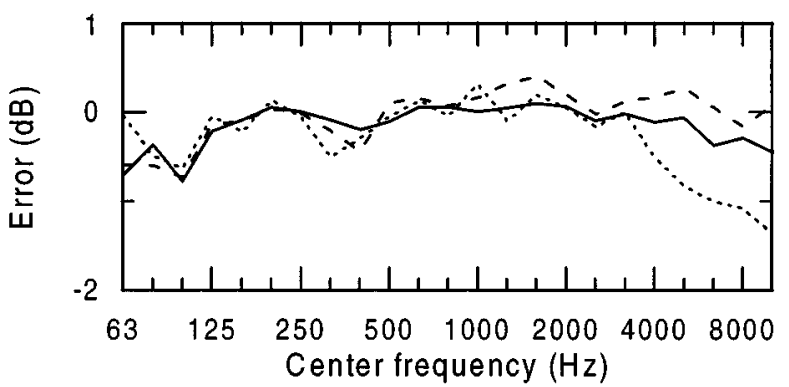

FIG. 11. Deviations between sound power measurements with an intensity probe with $\frac{1}{2}$-in. microphones and a $12-\mathrm{mm}$ spacer under three conditions and a sound power measurement with a probe with $\frac{1}{4}$-in. microphones and a 6-mm spacer under the most favorable of these conditions. — , reverberation room, no extraneous noise, ---, reverberation room, diffuse noise from distant source; $\cdots$, reverberation room, noise from source close to measurement surface.

probe so as to taper the $\frac{1}{2}$-in. microphones smoothly had no appreciable influence in sound power determination.

\section{CONCLUSIONS}

A numerical and experimental study of the performance of sound intensity probes with the microphones in the usual face-to-face arrangement has demonstrated that the optimum length of the spacer between the microphones is about one microphone diameter. With this geometry, diffraction effects tend to compensate for the finite difference approximation error inherent in the measurement principle, which means that the operational frequency range of the probe is significantly larger than hitherto believed.

A practical consequence is that a sound intensity probe with $\frac{1}{2}$-in. microphones separated by a $12-\mathrm{mm}$ spacer can cover the frequency range up to $10 \mathrm{kHz}$.

\section{APPENDIX: DIFFRACTION EFFECTS IN A PLANE STANDING WAVE FIELD}

Let $\hat{p}_{1}$ and $\hat{p}_{2}$ be the pressures as sensed by the microphones of a sound intensity probe in a plane progressive wave of axial incidence. If the intensity probe is symmetric about the spacer, it follows from the principle of superposition that the corresponding pressures are

$$
\begin{aligned}
& \hat{p}_{1}^{i}=\hat{p}_{1}+R \hat{p}_{2}, \\
& \hat{p}_{2}^{i}=\hat{p}_{2}+R \hat{p}_{1},
\end{aligned}
$$

in the interference field composed by the plane wave and another plane wave propagating in the opposite direction, where the latter wave is modified in amplitude and phase by the complex factor $R$. Therefore the indicated intensity in the standing wave field is

$$
\begin{aligned}
\hat{I}_{r}^{i} & =\frac{\operatorname{Im}\left\{\hat{p}_{1}^{i}\left(\hat{p}_{2}^{i}\right)^{*}\right\}}{2 \rho c k \Delta r} \\
& =\frac{\operatorname{Im}\left\{\left(\hat{p}_{1}+R \hat{p}_{2}\right)\left(\hat{p}_{2}^{*}+R^{*} \hat{p}_{1}^{*}\right)\right\}}{2 \rho c k \Delta r} \\
& =\frac{\operatorname{Im}\left\{\hat{p}_{1} \hat{p}_{2}^{*}+|R|^{2} \hat{p}_{1}^{*} \hat{p}_{2}\right\}}{2 \rho c k \Delta r}+\frac{\left|\hat{p}_{2}\right|^{2}-\left|\hat{p}_{1}\right|^{2}}{4 \rho c k \Delta r} 2|R| \sin \theta \\
& =\hat{I}_{r}\left(1-|R|^{2}\right)-2 \hat{J}_{r}|R| \sin \theta,
\end{aligned}
$$

where $\hat{I}_{r}$ is the indicated intensity (subject to diffraction) in the plane progressive wave, $\hat{J}_{r}$ is the corresponding indicated reactive intensity (which in this case is the result of diffraction-there is no reactive part in a plane wave), ${ }^{29}$ and $\theta$ is the phase angle of $R$. (The phase angle $\theta$ corresponds to the distance between the position of the probe and a position where the pressure assumes a maximum or a minimum value.) Since the true intensity in the standing wave field is

$$
I_{r}^{i}=I_{r}\left(1-|R|^{2}\right),
$$

where $I_{r}$ is the true intensity in the plane progressive wave, it can be seen that, surprisingly, the relative diffraction error is the same as in the plane wave in the standing wave at positions where the pressure assumes maximum and minimum values.

Equation (A2) is also valid at the position halfway between two interfering monopole sources in free space; Eq. (A3), however, is not.

${ }^{1}$ F. J. Fahy, Sound Intensity (Spon, London, 1995), 2nd ed. See sections 5.2, 5.6, and 6.2.

${ }^{2}$ G. Rasmussen and M. Brock, "Acoustic intensity measurement probe," in Proceedings Recent Developments in Acoustic Intensity Measurement, edited by M. Bockhoff (Senlis, France, 1981), pp. 81-88.

${ }^{3}$ F. J. Fahy and S. J. Elliott, "Practical considerations in the choice of transducers and signal processing techniques for sound intensity measurements," in Proceedings Recent Developments in Acoustic Intensity Measurement, edited by M. Bockhoff (Senlis, France, 1981), pp. 37-44.

${ }^{4}$ E. Frederiksen and O. Schultz, "Pressure microphones for intensity measurements with significantly improved phase properties," Brüel \& Kjær Tech. Rev. 4, 11-23 (1986).

${ }^{5}$ G. Pavić, "Measurement of sound intensity,' J. Sound Vib. 51, 533-545 (1977).

${ }^{6}$ J. K. Thompson and D. R. Tree, "Finite difference approximation error in acoustic intensity measurements," J. Sound Vib. 75, 229-238 (1981).

${ }^{7}$ U. S. Shirahatti and M. J. Crocker, "Two-microphone finite difference approximation errors in the interference fields of point dipole sources,' $\mathrm{J}$. Acoust. Soc. Am. 92, 258-267 (1992).

${ }^{8}$ S. Gade, "Sound intensity. Part I Theory,"' Brüel \& Kjær Tech. Rev. 3, 3-39 (1982)

${ }^{9}$ F. Jacobsen, "Sound intensity measurements at low levels," J. Sound Vib. 166, 195-207 (1993).

${ }^{10}$ F. Jacobsen and E. S. Olsen, "The influence of microphone vents on the performance of sound intensity probes," Appl. Acoust. 41, 25-45 (1994).

${ }^{11}$ A. C. Balant, G. C. Maling, Jr., and D. M. Yeager, "Measurement of blower and fan noise using sound intensity techniques," Noise Control Eng. J. 33, 77-88 (1989).

${ }^{12} \mathrm{~J}$. Tichy, "Some effects of microphone environment on intensity measurements," in Proceedings Recent Developments in Acoustic Intensity Measurement, edited by M. Bockhoff (Senlis, France, 1981), pp. 25-30.

${ }^{13} \mathrm{G}$. Krishnappa, "Interference effects in the two microphone technique of acoustic intensity measurements," Noise Control Eng. J. 21, 126-135 (1983).

${ }^{14}$ G. Krishnappa, "Scattering/diffraction effects in the two-microphone technique of measuring sound intensity at sound incidence angles other than $0^{\circ}$," Noise Control Eng. J. 22, 96-102 (1984). 
${ }^{15}$ P. S. Watkinson and F. J. Fahy, "Characteristics of microphone arrangements for sound intensity measurement,' J. Sound Vib. 94, 299-306 (1984).

${ }^{16}$ E. Frederiksen and M. Piil, "Characteristics of microphone pairs and probes for sound intensity measurements,', Brüel \& Kjær, Nærum, Report BA0077-11 (1987).

${ }^{17}$ J. C. Rébillat and S. Rifai, "Détermination expérimentale de la diffraction acoustique autour de sondes intensimétriques,' Acustica 70, 83-88 (1990).

${ }^{18}$ J. C. Rébillat, "Estimation numérique de la diffraction acoustique autour de sondes intensimétriques,'” Acustica 63, 203-210 (1987).

${ }^{19}$ B. B. Baker and E. T. Copson, The Mathematical Theory of Huygen's Principle (Oxford U.P., Oxford, 1953), 2nd ed.

${ }^{20}$ A. F. Seybert, B. Soenarko, F. J. Rizzo, and D. J. Shippy, “'An advanced computational method for radiation and scattering of acoustic waves in three dimensions,', J. Acoust. Soc. Am. 77, 362-368 (1985).

${ }^{21}$ V. Cutanda, P. M. Juhl, and F. Jacobsen, "The influence of thin shapes and narrow gaps on the convergence of boundary element formulations,", submitted to J. Acoust. Soc. Am.

${ }^{22}$ P. Juhl, "A numerical investigation of standard condenser microphones,", J. Sound Vib. 177, 433-446 (1994).
${ }^{23}$ O. Roth, “A sound intensity real-time analyzer,'” in Proceedings Recent Developments in Acoustic Intensity Measurement, edited by M. Bockhoff (Senlis, France, 1981), pp. 69-74.

${ }^{24}$ Anon.: Product Data. Sound Intensity Probes Types 3520, 3545, 3547, 3548. Sound Intensity Microphone Pairs Types 4178, 4181 (Brüel \& Kjær, Nærum, 1991).

${ }^{25}$ S. Gade and K. B. Ginn, "Design of a new robust sound intensity probe," in Proceedings of the Third International Congress on Air-and StructureBorne Sound and Vibration, edited by M. J. Crocker (Montréal, Canada, 1994), pp. 1037-1044.

${ }^{26}$ IEC International Standard 1043, "Electroacoustics-Instruments for the measurement of sound intensity-Measurements with pairs of pressure sensing microphones", (1993).

${ }^{27}$ ANSI S1.12-1994, “American National Standard Instruments for the measurement of sound intensity" (American National Standards Institute, New York, 1994).

${ }^{28} \mathrm{~F}$. Jacobsen, "A note on the accuracy of phase compensated intensity measurements,', J. Sound Vib. 174, 140-144 (1994).

${ }^{29}$ F. Jacobsen, "Active and reactive, coherent and incoherent sound fields,", J. Sound Vib. 130, 493-507 (1989). 